TRANSACTIONS OF THE

AMERICAN MATHEMATICAL SOCIETY

Volume 352, Number 9, Pages 4095-4111

S 0002-9947(00)02473-9

Article electronically published on April 19, 2000

\title{
A REGULAR SPACE WITH A COUNTABLE NETWORK AND DIFFERENT DIMENSIONS
}

\author{
GEORGE DELISTATHIS AND STEPHEN WATSON
}

\begin{abstract}
In this paper, we construct a regular space with a countable network (even the union of countably many separable metric subspaces) in which ind and dim do not coincide under the assumption of the continuum hypothesis $(\mathrm{CH})$. This gives a consistent negative answer to a question of A.V. Arhangel'skiı.
\end{abstract}

\section{INTRODUCTION}

It is well-known that all three dimension functions $\operatorname{dim} X$, ind $X$ and $\operatorname{Ind} X$ coincide when $X$ is a separable metric space (see [3]). At the International Congress of Mathematicians in Nice in 1970, Arhangel'skiu asked whether this is also true for (regular) continuous images of separable metric spaces (problem 4, page 24 in [1]) and Gruenhage states this as question 10.2 in his survey paper [8]. Such images are precisely the spaces with a countable network.

These spaces are hereditarily Lindelöf, so (by Corollary 5.3.11 in [4, and Theorems 2.1.4. and 2.4.4. in [3), they satisfy ind $X=\operatorname{Ind} X$. Since we also have that $\operatorname{dim} X \leq$ ind $X$ for $X$ Lindelöf and that $\operatorname{dim} X=0$ iff $\operatorname{Ind} X=0$ for $X$ normal (see [3]), we get that any potential counterexample $X$ should have $\operatorname{dim} X \geq 1$ and ind $X=\operatorname{Ind} X \geq 2$. Our space $X$ will have $\operatorname{dim} X=1$ and $\operatorname{ind} X=\operatorname{Ind} X \geq 2$ and, in fact, ind $B d(U) \geq 1$ for all non-empty open subsets $U$ of $X$ that are not dense in $X$. In particular, then, ind $_{x} X \geq 2$ for all $x \in X$.

In [11] (or see [13]), Nagami discussed the dimension theory of spaces $X$ that are paracompact, Hausdorff and the countable union of metrizable closed subspaces $\left(X_{n}\right)_{n \in \omega}$. In these spaces, $\operatorname{dim} X=\operatorname{Ind} X$. In [12], Oka showed that $\operatorname{dim} X=\operatorname{Ind} X$ if $X$ is paracompact, perfectly normal and the finite union of metric subspaces and asked whether $\operatorname{dim} X=\operatorname{Ind} X$ if $X$ is paracompact, perfectly normal and the countable union of metric subspaces. So Oka's question has been answered here in the negative (under $\mathrm{CH}$ ).

All this is accomplished with an inductive construction of length $\omega_{1}$ (using $\mathrm{CH}$ ) applying a result of Kuratowski (see [9]), which is presented using the method of resolutions. We refine the topology, making sure that each time we do this, we preserve the fact that this space is a particular countable union of separable metric subspaces and that the covering dimension ( $\mathrm{dim}$ ) does not increase. Since $X$ is then a union of countably many separable metric subspaces, $X$ has a countable network. Let us proceed to the details of the argument.

Received by the editors February 16, 1996 and, in revised form, November 18, 1998.

2000 Mathematics Subject Classification. Primary 54F45, 54E20; Secondary 54A25, 54G20.

(C)2000 American Mathematical Society 


\section{The METhOD OF RESOLUtions}

The method of resolutions was invented and used by Fedorčuk to construct compact spaces with interesting properties (see for instance [5], 6]). The second author has written a survey paper on the subject (see [14]), where the interested reader will find many more references.

The method of resolutions is described as follows:

Let $X$ be a space and for each $x \in X$, let $Y_{x}$ be a space and let $f_{x}: X \backslash\{x\} \longrightarrow Y_{x}$ be a continuous mapping; we let $Z=\bigcup\left\{\{x\} \times Y_{x}: x \in X\right\}$ and if $z=(x, y) \in$ $\{x\} \times Y_{x}$ we define $U \otimes V=\{x\} \times V \cup \bigcup\left\{\left\{x^{\prime}\right\} \times Y_{x^{\prime}}: x^{\prime} \in U \cap f_{x}^{-1}(V)\right\}$, where $x \in U, U$ is open in $X$ and $y \in V, V$ is open in $Y_{x}$ to be a local base at $z$.

We observe that if $S$ is a subspace of $X$, the resolution space of the subspace $S$ is a subspace of $Z$ when we use the restrictions of the mappings $f_{x}$ to $S \backslash\{x\}$. It is also easy to check that the projection map $\pi: Z \longrightarrow X$ defined by $\pi(x, y)=x$ for $y \in Y_{x}$ is continuous and onto. The method of resolutions can be used to refine a topology on a given space $X$. With $X, Y_{x}, f_{x}$ as before, for all $x \in X$ pick a point $y_{x} \in Y_{x}$ and let $Z^{\prime}=\left\{\left(x, y_{x}\right): x \in X\right\} \subseteq Z$, where $Z$ is the resolution space as before. We observe that $\pi \uparrow Z^{\prime}: Z^{\prime} \longrightarrow X$ is $1-1$, onto and continuous, so if we give $X$ the quotient topology of $\pi \uparrow Z^{\prime}$, the original topology of $X$ is refined and a basic open set containing $x$ will be $\{x\} \cup\left(U \cap f_{x}^{-1}(V)\right)$, where $x \in U$ and $U$ is open in $X, y_{x} \in V$ and $V$ is open in $Y_{x}$. It is now obvious that if $Y_{x}$ is a singleton, then the topology is not refined at the point $x \in X$.

This process can be iterated; more precisely, if $\alpha$ is a successor ordinal, we refine the topology as we described before and if $\alpha$ is a limit ordinal, we take the inverse limit of $\left(X, \tau_{\beta}\right)$ for all $\beta<\alpha$. It follows that $\tau_{\alpha}=\sup \left\{\tau_{\beta}: \beta<\alpha\right\}$, since the mapping $j: \lim \left\{X_{\beta}: \beta<\alpha\right\} \longrightarrow\left(X, \sup \left\{\tau_{\beta}: \beta<\alpha\right\}\right)$ defined by $j(x, x, \ldots)=x$ is a homeomorphism (a net $\left(x_{i}\right)_{i \in I}$ converges to $x$ in $\sup \left\{\tau_{\beta}: \beta<\alpha\right\}$ iff for all $\beta<\alpha$, for all $G \in \tau_{\beta}$, there exists $i_{0} \in I$ so that $x_{i} \in G$ for all $i \geq i_{0}$; this is equivalent to the statement that $x_{i} \rightarrow x$ in each $\tau_{\beta}$ and we have verified that $j$ is indeed a homeomorphism).

If we iterate this process finitely many times, a basic open set containing $x \in X$ will be of the form $\{x\} \cup\left(U \cap f_{1, x}^{-1}\left(G_{1}\right) \cap \ldots \cap f_{k, x}^{-1}\left(G_{k}\right)\right)$, so, whenever the functions $f_{x}$ are continuous when $X$ has the original topology, the order of resolving is not significant, i.e. the resulting spaces are homeomorphic. In the general case, i.e. for any ordinal $\alpha$, we will have that $\alpha=\lambda+n$, where $\lambda$ is a limit ordinal and $n \in \omega$, so a basic open set containing $x \in X$ will be of the same form (as before) and it follows immediately (by induction) that the order of resolving is again not significant.

In [14, it is proved that if $X$ and all $Y_{x}$ 's are compact Hausdorff spaces, then so is $Z$; in the same paper, the fact that if $X$ and all $Y_{x}$ 's are regular spaces, then this is also true for $Z$, is used but not proved. Therefore, for the sake of completeness, we state and prove the following

Proposition 1.1. Let $X$ be a regular space and for each $x \in X$, let $Y_{x}$ be a regular space and let $f_{x}: X \backslash\{x\} \longrightarrow Y_{x}$ be a continuous mapping. Then $Z=\bigcup\left\{\{x\} \times Y_{x}\right.$ : $x \in X\}$ is a regular space with the resolution topology.

Proof. Let $z_{0}=\left(x_{0}, y_{0}\right) \in Z$ and let $U \otimes V$ be a basic neighbourhood of $z_{0}$, where $x_{0} \in U$ and $y_{0} \in V, U$ is open in $X$ and $V$ is open in $Y_{x_{0}}$. Since $X$ and $Y_{x_{0}}$ are regular spaces, we can find open sets $W, G$ in $X, Y_{x_{0}}$ respectively, such that $x_{0} \in W \subseteq \bar{W} \subseteq U$ and $y_{0} \in G \subseteq \bar{G} \subseteq V$. 
We will show that $z_{0}=\left(x_{0}, y_{0}\right) \in W \otimes G \subseteq \overline{W \otimes G}^{Z} \subseteq K=\left\{x_{0}\right\} \times \bar{G} \cup \bigcup\left\{\left\{x^{\prime}\right\} \times\right.$ $\left.Y_{x^{\prime}}: x^{\prime} \in \bar{W} \cap f_{x_{0}}^{-1}(\bar{G})\right\} \subseteq U \otimes V$ and that will complete the proof. The only nontrivial inclusion is $\overline{W \otimes G}^{Z} \subseteq K$; it suffices to show that $K$ is closed, or that $Z \backslash K$ is open. We distinguish two cases:

Case 1: Let $z=(x, y) \notin K, x=x_{0}$; we then have that $y \notin \bar{G}$, so $y \in A, A$ is open in $Y_{x_{0}}$ and $A \cap \bar{G}=\emptyset$. But then $z=\left(x_{0}, y\right) \in X \otimes A=\left\{x_{0}\right\} \times A \cup \bigcup\left\{\left\{x^{\prime}\right\} \times Y_{x^{\prime}}\right.$ : $\left.x^{\prime} \in f_{x_{0}}^{-1}(A)\right\}$ which is open and misses $K$, so we are done.

Case 2: Let $z=(x, y) \notin K, x \neq x_{0}$; we then have that $x \notin \bar{W} \cap f_{x_{0}}^{-1}(\bar{G})=F$ and $F$ is closed in $X \backslash\left\{x_{0}\right\}$. We get that $F \cup\left\{x_{0}\right\}$ is closed in $X$ and we have that $z=(x, y) \in\left(F \cup\left\{x_{0}\right\}\right)^{c} \otimes Y_{x}$, which is an open set that misses $K$.

Proposition 1.2. With the same notation as in proposition 1.1 supposing that $\left|\left\{x \in X:\left|Y_{x}\right| \geq 2\right\}\right|=\kappa$, we have that $w(Z) \leq \max \left\{w(X), \kappa \cdot \sup \left\{w\left(Y_{x}\right): x \in\right.\right.$ $X\}\}=\lambda$.

Proof. Recall that a basis for $Z$ is all sets $U \otimes V, U$ open in $X, V$ open in $Y_{x}$ for some $x \in X$. If $\left|Y_{x}\right|=1$, then $U \otimes V=\bigcup\left\{\left\{x^{\prime}\right\} \times Y_{x^{\prime}}: x^{\prime} \in U\right\}$, so we only need $w(X) \leq \lambda$ many such sets; the rest of the $Y_{x}$ 's are $\leq \lambda$ many, so (as $w\left(Y_{x}\right) \leq \lambda$ for all $x \in X$ and $w(X) \leq \lambda$ ) we only need $\leq \lambda$ more open sets and we get that $w(Z) \leq \lambda$

Corollary 1.3. With the same notation as in proposition 1.1, supposing that $X$ is a separable metric space, $Y_{x}$ is a separable metric space for all $x \in X$ and $\left|\left\{x \in X:\left|Y_{x}\right| \geq 2\right\}\right| \leq \aleph_{0}, Z$ is a separable metric space.

Proof. This is immediate from propositions 1.1 1.2 and the well-known fact that a space $S$ is a separable metric iff $S$ is regular and second countable.

\section{The eXample of Kuratowski}

Motivated by [9] (see also [3], exercise 1.2.E.), we have the following result:

Proposition 2.1. Suppose $(S, \tau)$ is a space such that every dense in itself closed subset of $S$ is uncountable and let $D$ be a countable subset of $S$. Suppose $\sigma$ is a topology on $S$ that is smaller than $\tau$ and $f: S \longrightarrow[-1,1], h: D \longrightarrow \omega$ which satisfy the following:

(i) For each $x \in D$, there exist two sequences $\left(x_{m}^{\prime}\right)_{m \in \omega},\left(x_{m}^{\prime \prime}\right)_{m \in \omega} \in D^{\omega}$ that converge to $x$ in $\sigma$ so that the following hold for every $m \in \omega$ :

1. $h\left(x_{m}^{\prime}\right)=h\left(x_{m}^{\prime \prime}\right)=h(x)+1$,

2. $f\left(x_{m}^{\prime}\right)-2^{-h\left(x_{m}^{\prime}\right)}=f(x)$,

3. $f\left(x_{m}^{\prime \prime}\right)+2^{-h\left(x_{m}^{\prime \prime}\right)}=f(x)$,

(ii) For each $x \in D, f(x)+2^{-h(x)}$ is the upper limit $f^{h}(x)$ of $f$ at $x$ in $\sigma$ and $f(x)-2^{-h(x)}$ is the lower limit $f_{h}(x)$ of $f$ at $x$ in $\sigma$,

(iii) $f:(S, \sigma) \longrightarrow[-1,1]$ is continuous at $x$ if $x \notin D$,

(iv) For each $x \in D$, there exist $\left(x_{m_{i}}^{\prime}\right)_{i \in \omega},\left(x_{m_{j}}^{\prime \prime}\right)_{j \in \omega}$ (subsequences of $\left(x_{m}^{\prime}\right)_{m \in \omega}$, $\left(x_{m}^{\prime \prime}\right)_{m \in \omega}$, respectively) that converge to $x$ in $\tau$. $\emptyset$.

Suppose $H$ is a countable subset of $G r(f)$, the graph of $f$, such that $p_{1}(H) \cap D=$

Then $I=G r(f) \backslash H \subseteq(S, \tau) \times[-1,1]$ is not 0 -dimensional at $(x, f(x))$ for all $x \in D$. 
Proof. Let $J=G r(f) \cup \bigcup_{x \in D}\{x\} \times\left[f_{h}(x), f^{h}(x)\right]$ and denote $(x, f(x)),\left(x, f^{h}(x)\right)$, $\left(x, f_{h}(x)\right)$ by $z, \bar{z}, \underline{z}$ respectively. In this proof, $x$ 's and $z$ 's (or $\bar{z}$ 's, $\underline{z}$ 's) will correspond to one another if they have the same index.

Now $J$ is closed in $(S, \sigma) \times[-1,1]$ (using (ii) and (iii)), so $J$ is also closed in $(S, \tau) \times[-1,1]$. We now show that $\operatorname{ind}_{I} z>0$ for all $z \in I$ such that $x \in D$; suppose this is not the case and let $i n d_{I} z_{0}=0$ for some $z_{0} \in I, x_{0} \in D$.

We claim that there exists $C \subseteq J \backslash I$, a closed subset of $(S, \tau) \times[-1,1]$ which separates $J$ in two open sets $A, B$ such that $z_{0} \in A$ and $\overline{z_{0}} \in B$ (or $\underline{z}_{0} \in B$ ). Indeed, let $V, W$ be disjoint open sets in $(S, \tau) \times[-1,1]$ such that $z_{0} \in V, \overline{z_{0}} \in W$; since $i n d_{I} z_{0}=0$, we apply exercise 1.2.A in [3] (or see [13], lemma 4.16) to find an open set $U \ni z_{0}$ such that $U \subseteq V$ and $I \cap B d(U)=\emptyset$ and we observe that $z_{0} \in U \cap J=A, \overline{z_{0}} \in B=W \cap J \subseteq J \backslash \bar{V} \subseteq J \backslash \bar{U}$, so $C=J \cap B d(U) \subseteq J \backslash I$ works.

We observe that $C$ is closed in $J$, so in $(S, \tau) \times[-1,1]$. Hence $p_{1}(C)$ is closed (being the projection of a closed set along the compact factor $[-1,1]$ ) and $p_{1}(C) \subseteq$ $D \cup p_{1}(H)$, so $p_{1}(C)$ is countable and, then, by our hypothesis, $p_{1}(C)$ does not contain any dense in itself subset.

Let $E=\{x \in S: C$ separates $z$ and $\bar{z}$ or $z$ and $\underline{z}\}$; the intervals $z \bar{z}, \underline{z} z$ are connected, so for all $x \in E, C \cap \underline{z} \bar{z} \neq \emptyset$, hence $E \subseteq p_{1}(C)$ and $E$ has an isolated point $x\left(E \neq \emptyset\right.$ as $\left.x_{0} \in E\right)$. Without loss of generality, one may assume that $z \in A$ and $\bar{z} \in B$ (otherwise, the proof is similar). Using equalities (1), (2), (3) of (i), it is easy to see that the following equations are true for all $m \in \omega$ :

(4) $f^{h}\left(x_{m}^{\prime}\right)=f^{h}(x), f_{h}\left(x_{m}^{\prime}\right)=f(x)$,

(5) $f^{h}\left(x_{m}^{\prime \prime}\right)=f(x), f_{h}\left(x_{m}^{\prime \prime}\right)=f_{h}(x)$.

By (4) and (iv), we have that $z_{m_{i}}^{\prime} \in A$ and $\overline{z_{m_{i}}^{\prime}} \in B$ for all sufficiently large $i$. Since $D \cap p_{1}(H)=\emptyset$, we get that $\frac{m_{i}}{z_{m_{i}}^{\prime}} \in I$ so $z_{m_{i}}^{\prime} \notin C$ and $z_{m_{i}}^{\prime} \in A$ or $z_{m_{i}}^{\prime} \in B$. In

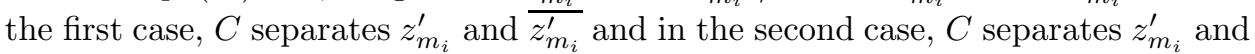
$\underline{z_{m_{i}}^{\prime}}$. Therefore, $x_{m_{i}}^{\prime} \in E$ and $x$ is not an isolated point of $E$ (recall that $x_{m_{i}}^{\prime} \underset{\tau}{\longrightarrow} x$ ); this contradiction completes the proof.

What Kuratowski actually showed in [9] (see also [3], exercise 1.2.E.), is the following:

Lemma 2.2. Suppose $D=\left\{d_{n}: n \in \omega\right\}$ is the set of endpoints of the open intervals removed during the construction of the Cantor set (so $D$ is a countable dense subset of the Cantor set) and suppose $\sigma$ is the Euclidean topology of $2^{\omega}$. There exist $f: 2^{\omega} \longrightarrow[-1,1]$ and $h: D \longrightarrow \omega$ and two sequences $\left(x_{m}^{\prime}\right)_{m \in \omega},\left(x_{m}^{\prime \prime}\right)_{m \in \omega} \in D^{\omega}$ that converge to $x$ in $\sigma$ that satisfy conditions (i), (ii) and (iii) of proposition 2.1. Moreover, for each $n \in \omega$, the oscillations osc $(f)\left(d_{n}\right)$ of $f$ at the points $d_{n}$ converge to 0 .

This fact allows us to give an alternate description of the Kuratowski example; we first state and prove the following

Lemma 2.3. For every $x \in D$, there exists a continuous function $g_{x}: 2^{\omega} \backslash\{x\} \longrightarrow$ $[-1,1]$ such that $\lim _{x^{\prime} \rightarrow x, x^{\prime} \in 2^{\omega}}\left|g_{x}\left(x^{\prime}\right)-f\left(x^{\prime}\right)\right|=0$.

Proof. Without loss of generality, one may suppose that $x$ is a right endpoint, i.e. there exists $\delta>0$ such that $[x, x+\delta) \cap 2^{\omega}=\{x\}$. Using $\operatorname{osc}(f)\left(d_{n}\right) \rightarrow 0$, we get that $x$ has a neighbourhood base of clopen sets $\left(V_{n} \cap 2^{\omega}\right)_{n \in \omega}$ with $V_{n}=\left(x_{n}, x_{n+1}\right) \cup$ $\left(x_{n+1}, x_{n+2}\right) \cup \ldots \cup\{x\}, x_{n} \notin 2^{\omega}, x_{n} \nearrow x$ and $\operatorname{osc}(f)(t)<\frac{1}{2^{n}} \forall t \in V_{n} \cap 2^{\omega} \backslash\{t\}$. Fix 
some $n \in \omega$ and for all $t \in\left(x_{n}, x_{n+1}\right) \cap 2^{\omega}$ let $V_{t}$ be a clopen neighbourhood of $t$ with $\operatorname{diam} f\left(V_{t}\right)<\frac{1}{2^{n}}$. Applying compactness and 0-dimensionality of $\left(x_{n}, x_{n+1}\right) \cap 2^{\omega}$, we get a finite disjoint open (hence clopen) refinement of this cover; so, if $A$ is a member of this finite cover, we pick $t$ such that $A \subseteq V_{t}$ and we define $g_{x}\left(x^{\prime}\right)=f(t)$ for all $x^{\prime} \in A$. By our definition, $g_{x}$ is constant (hence continuous) on each such $A$, so it is continuous on each $\left(x_{n}, x_{n+1}\right)$; let $g_{x}\left(x^{\prime}\right)=0$ if $x^{\prime}<x_{0}$ or $x^{\prime}>x$ and observe that $g_{x}$ is continuous. Finally, $\lim _{x^{\prime} \rightarrow x}\left|g_{x}\left(x^{\prime}\right)-f\left(x^{\prime}\right)\right|=0$ by the definition of $g_{x}$ since we chose the open sets $V_{t}$ so that $\operatorname{diam} f\left(V_{t}\right)<\frac{1}{2^{n}}$.

We now can describe Kuratowski's example as follows:

Proposition 2.4. Let $f$ be the Kuratowski function. Let $Z$ be the space constructed by resolving each $x \in 2^{\omega}$ as follows: if $x \in D$, resolve $x$ into $[-1,1]$ using the continuous function $g_{x}$ from lemma 2.3 and if $x \notin D$ use the constant function $g_{x}\left(x^{\prime}\right)=f(x)$. The space $G r(f)$ is canonically embedded as subspace of $Z$.

Proof. We will show that $\left(x_{n}, f\left(x_{n}\right)\right)$ converges to $(x, f(x))$ in the resolution topology iff the same thing happens in the graph topology.

Indeed, suppose that $\left(x_{n}, f\left(x_{n}\right)\right) \rightarrow(x, f(x))$ in the resolution topology. We get that $x_{n} \rightarrow x$ (the projection is continuous) and we only need to show that $f\left(x_{n}\right) \rightarrow f(x)$ if $x \in D$, as $f$ is continuous at $x$ if $x \notin D$.

Recalling that a basic neighbourhood of $(x, f(x))$ is

$$
V=\{(x, f(x))\} \cup \bigcup\left\{\left(x^{\prime}, f\left(x^{\prime}\right)\right): x^{\prime} \in U \cap g_{x}^{-1}(W)\right\}
$$

( $U$ is open, $x \in U, W$ is open and $f(x) \in W$ ) we get that, for sufficiently large $n$, $g_{x}\left(x_{n}\right) \in W$, so $g_{x}\left(x_{n}\right) \rightarrow f(x)$ and $f\left(x_{n}\right) \rightarrow f(x)$ by the construction of $g_{x}$.

Conversely, suppose that $x_{n} \rightarrow x$ and that $f\left(x_{n}\right) \rightarrow f(x)$. By the construction of $g_{x}$, we know $g_{x}\left(x_{n}\right) \rightarrow f(x)$, and so that, for sufficiently large $n, g_{x}\left(x_{n}\right) \in W$ and $x_{n} \in U$. Thus $\left(x_{n}, f\left(x_{n}\right)\right) \in V$ and we are done.

\section{The construction}

Our plan is to iterate the process just described so that we will get a regular space $X$ with a countable network such that $\operatorname{dim} X=1$ and $i n d B d(U) \geq 1$ if $U$ is a non-empty open subset of $X$ that is not dense (therefore ind $X=\operatorname{Ind} X \geq 2$ ). In order to accomplish this, it will be shown that a separator in $[0,1]^{2}$ contains a Cantor set of a special kind, which we then resolve applying the Kuratowski method as we have described it.

In the discussion to follow, a rational segment is a segment joining two points with rational coordinates and if we do not explicitly refer to a topology, we mean that we refer to the usual Euclidean topology.

Proposition 3.1 $(\mathrm{CH})$. For each $x \in[0,1]^{2}$, there exists a family of sequences of pairwise disjoint closed squares $\mathcal{S}(x)=\left\{\left\{B_{i, x}^{\alpha}: i \in \omega\right\}: \alpha<\omega_{1}\right\}$ such that $\operatorname{diam} B_{i, x} \rightarrow 0, d\left(x, B_{i, x}\right) \rightarrow 0$ and the following hold:

(I) For all $\alpha<\omega_{1}$, for all sequences $\left(x_{i}\right)_{i \in \omega}$ such that $x_{i} \rightarrow x$ and $x_{i} \notin \bigcup_{j \in \omega} B_{j, x}^{\alpha}$ and for all sequences of open sets $\left(V_{i}\right)_{i \in \omega}$ such that $x_{i} \in V_{i}$, there exists $S=\left\{B_{i}\right.$ : $i \in \omega\} \in \mathcal{S}(x)$ such that $x_{i} \in \operatorname{Int}\left(B_{i}\right) \subseteq B_{i} \subseteq V_{i}$ and $\bigcup S \cap \bigcup_{i \in \omega} B_{i, x}^{\alpha}=\emptyset$ (note that in order to achieve (I), a family of size continuum is needed; this is an apparently essential use of $\mathrm{CH}$ or some set-theoretic hypothesis, at any rate), 
(II) Suppose $x \in[0,1]^{2}$. For any $S, S^{\prime} \in \mathcal{S}$, there is $S^{\prime \prime} \in \mathcal{S}$ which contains a tail of both $S$ and $S^{\prime}$. Furthermore, for any sequence $\left(S_{i}\right)_{i \in \omega}$ such that $S_{i} \in \mathcal{S}(x)$ for all $i \in \omega$ and $S_{j}$ contains a tail of $S_{i}$ for all $j \geq i$, there exists $S \in \mathcal{S}(x)$ such that $S$ contains a tail of each $S_{i}$.

(III) Furthermore, suppose $U$ is a non-empty Euclidean open set in $[0,1]^{2}$ that is not dense, let $S=B d(U)$ and let $V=\bar{U}^{c} \neq \emptyset$. Note that $[0,1]^{2} \backslash S=U \cup V$. Suppose also that $p, q \in Q^{2}$ (Q will denote the set of rationals) so that $p \in U$ and $q \in V$. Let $R$ be the rational segment joining $p$ and $q$. Let $U^{\prime}$ and $V^{\prime}$ be squares of equal size centered at $p$ and $q$, respectively, contained in $U$ and $V$, respectively, and each having one opposite pair of sides parallel to $R$. Let $M^{\prime}$ be the rectangle which has as opposite sides the sides of $U^{\prime}$ and $V^{\prime}$ which intersect $R$. Suppose also that $[0,1]^{2}$ has a (stronger than the Euclidean) separable metric topology $\tau$ such that all but countably many points of $[0,1]^{2}$ have a Euclidean local base and suppose that, if $x$ is one of these exceptional countably many points, then $x$ belongs to a rational segment and has a local base so that, for any element $P$ of the base, there is $\alpha<\omega_{1}$ such that $P$ contains an open square minus the union of a tail of the sequence $\left(B_{i, x}^{\alpha}\right)_{i \in \omega}$. Then, if $S$ separates $U^{\prime}, V^{\prime}$, then there exist $x \in R$, a sequence $x_{n} \rightarrow x$ and a sequence of rational segments $\left(R_{n}\right)_{n \in \omega}$ such that $x_{n} \in R_{n}, x_{n} \notin R$, $x_{n} \in M^{\prime} \cap S$ and $x_{n} \rightarrow x$ in $\tau$. In the case that $x$ is one of the countably many exceptional points, we will have that for all $n \in \omega, x_{n} \notin \bigcup_{i \in \omega} B_{i, x}^{\alpha}$.

Proof. First of all, we observe that if $\left(B_{i, x}\right)_{i \in \omega}$ is as in the statement of the proposition, then $\left(B_{i, x}\right)_{i \in \omega} \rightarrow x$.

For each $x \in[0,1]^{2}$, let $\mathcal{S}(x)$ be the family of all sequences $\left(B_{i, x}\right)_{i \in \omega}$ consisting of pairwise disjoint closed squares $B_{i, x}$ whose diameter is $d_{i, x}$ such that $d\left(x, B_{i, x}\right)=$ $r_{i, x} \rightarrow 0$ and $\Sigma_{i \in \omega} \frac{d_{i, x}}{r_{i, x}}<\frac{1}{2}$. In particular, we have that $\frac{d_{i, x}}{r_{i, x}} \rightarrow 0$ and (using that $\left.r_{i, x} \rightarrow 0\right)$ we conclude that $d_{i, x} \rightarrow 0$ and that $\left(B_{i, x}\right)_{i \in \omega} \rightarrow x$.

(I) follows easily from the definition of $\mathcal{S}(x)$.

We prove (II). The basic observation is that if $j \geq i$, only finitely many squares of the sequence $S_{j}$ will intersect a square (other than itself) of the sequence $S_{i}$. (To see this, use our hypothesis that $\left(S_{i}\right)_{i \in \omega}$ is almost decreasing, and that each $S_{i}$ converges to $x$. We therefore get that, for all $j \in \omega$, only finitely many squares of the sequence $S_{j}$ will intersect a square (other than itself) of any sequence $S_{i}$ where $j \geq i$.

Using this observation, we pick a tail $T_{j}$ of each of the $S_{j}$ 's such that the sum of the tail of the corresponding series will be smaller than $2^{-(j+2)}$ and each square in the $j$-th tail will miss all previous squares in all previous tails. It is then clear that $S=\bigcup_{j \in \omega} T_{j}$ is as required.

We prove (III). First we prove:

Claim: Suppose $x$ is one of the countably many exceptional points. There exists a sequence of annuli $\left(A_{n, x}\right)_{n \in \omega}$ that converges to $x$ such that for all $n \in \omega, A_{n, x} \cap$ $\bigcup_{i \in \omega} B_{i, x}^{\alpha}=\emptyset$.

Proof of the claim: Let $x=\left(x_{0}, y_{0}\right)$ be one of these countably many points, let $\mathbb{R}$ denote the real numbers and let $H=\left\{x_{0}\right\} \times\left[y_{0}, \infty\right)$. Consider the map $f: \mathbb{R}^{2} \longrightarrow H$ defined by $f(z)=\left(x_{0}, y_{0}+d(x, z)\right)$. It suffices to show that the range of $f \uparrow \bigcup_{i \in \omega} B_{i, x}^{\alpha}$ misses a sequence of intervals approaching $x$.

We first observe that the range of this restriction cannot contain any set $I_{\delta}=$ $\{y \in H: \delta<d(x, y)<2 \delta\}$, where $\delta>0$. Indeed, by the triangle inequality, $d(f(z), f(w)) \leq d(z, w)$ for all $z, w$ in $\mathbb{R}^{2}$, therefore $\operatorname{diam} f\left(B_{i, x}^{\alpha}\right) \leq \operatorname{diam} B_{i, x}^{\alpha}=d_{i, x}$ 
and $r_{i, x}<2 \delta$ for any closed square $B_{i, x}^{\alpha}$ that satisfies $f\left(B_{i, x}^{\alpha}\right) \cap I_{\delta} \neq \emptyset$. Observe that there are only finitely many $i \in \omega$ such that $f\left(B_{i, x}^{\alpha}\right) \cap I_{\delta} \neq \emptyset$ and let this finite set be $F$. We will have that $\frac{1}{2 \delta} \Sigma_{i \in F} d_{i, x}<\Sigma_{i \in F} \frac{d_{i, x}}{r_{i, x}}<\Sigma_{i \in \omega} \frac{d_{i, x}}{r_{i, x}}<\frac{1}{2}$ and we get that $\Sigma_{i \in \omega} d_{i, x}<\delta$, so we verified that our observation is correct. Since $\delta$ was arbitrary, the proof of the claim is finished, since $I_{\delta} \backslash \operatorname{Range}\left(f \backslash \bigcup_{i \in \omega} B_{i, x}^{\alpha}\right)$ will be a non-empty open set.

Now let $U^{\prime}, S, V^{\prime}, M^{\prime}, R, p, q$ be as in the statement of the proposition, let $K=M^{\prime} \cap S$ (observe that $K$ separates $U^{\prime}$ and $V^{\prime}$ in $U^{\prime} \cup V^{\prime} \cup M^{\prime}$ ) and suppose that (III) is not true.

Let $A$ be the countable set of points of $R$ where we have refined the topology and let $Z=\bigcup\left\{K \cap R^{\prime}: R^{\prime}\right.$ is a rational segment, $\left.R^{\prime} \cap K \cap R=\emptyset\right\}$. We distinguish two subcases:

Case 1: $\bar{Z} \cap R \backslash A=B \neq \emptyset$.

Choose $x \in B$. Now there is a sequence $\left(x_{n}\right)_{n \in \omega}$ as in (III) that converges to $x$ in the Euclidean topology. But $x_{n} \rightarrow x$ in $\tau$ since $x \notin A$.

Case 2: Not case 1

We will have that $\bar{Z} \cap R=L \subseteq A$. In particular, $L$ is a countable, compact, metric space, therefore $L$ is 0 -dimensional and scattered. (Observe that $L \neq \emptyset$, since if $R_{n}$ is a sequence of rational segments that are parallel and converge to $R$ and $x_{n} \in R_{n} \cap K$, then some subsequence $\left(x_{n_{i}}\right)_{i \in \omega}$ of $\left(x_{n}\right)_{n \in \omega}$ will converge to a point $x \in \bar{Z} \cap R=L$.)

Since (III) fails, it follows that for each $x \in L$, there exists $V \ni x$ such that $V$ is open in $[0,1]^{2}$ and $Z \cap V \subseteq \bigcup_{i \in \omega} B_{i, x}^{\alpha}$, so, using the claim, we get that there exists a sequence of annuli $\left(A_{n, x}\right)_{n \in \omega}$ that converges to $x$ such that for all $n \in \omega, A_{n, x} \cap Z=\emptyset$.

We now observe that the points of $L$ that are of maximum scattered height $\alpha$ form a finite set $\left\{l_{1}, \ldots, l_{k}\right\}$, so one can find pairwise disjoint intervals $G_{1}, \ldots, G_{k} \subseteq R$ such that for all $i=1, \ldots, k, l_{i} \in G_{i}$. We now find annuli $A_{1}, \ldots, A_{k}$ (where each $A_{i}$ is $A_{n, l_{i}}$ for some $n$ ) such that for all $i=1, \ldots, k, \overline{A_{i}} \cap R \subseteq G_{i}$ and we observe that, for each annulus $A_{i}$, we can find a polygonal line $P_{i} \subseteq A_{i}$ (consisting of rational segments that are either parallel or perpendicular to $R$ ) that connects the two points of $\overline{A_{i}} \cap R$ that belong to the outer circle of $A_{i}$ (in the sequel, they are called endpoints). So $P_{i}$ therefore misses $R$ except for two half-intervals containing these two endpoints. Since $L$ is 0 -dimensional, we may also assume that the endpoints of these half-intervals are not in $L$.

Hence, there is an open subinterval $J_{i}$ of $R$ such that each $l_{i} \in J_{i}$, and so that the endpoints of $J_{i}$ are both not in $L$ (in particular, $J_{i} \cap L$ is clopen in $L$ ) and can be joined by a polygonal path $P_{i}^{\prime}$ that misses $K$, except possibly for the two endpoints. (Recall the construction of the $A_{i}$ 's and the definition of $Z$ ).

Now let $L_{1}=L \backslash \bigcup_{i=1, \ldots, k} J_{i}$ and suppose that $L_{1} \neq \emptyset$; we have that $L_{1}$ is clopen in $L$ and that its maximum scattered height is $\alpha_{1}<\alpha$. Since we suppose that (III) fails, we may repeat the argument we gave for $L$ to get $J_{i}^{\prime}$ 's that are disjoint from all $J_{i}$ 's, $L_{2}=L_{1} \backslash \bigcup_{i=1, \ldots, k^{\prime}} J_{i}^{\prime}$ and so on. But then we will have that there exists $i \in \omega$ such that $L_{i}=\emptyset$, since otherwise the maximum scattered heights of the $L_{i}$ 's would form an infinite decreasing sequence of ordinals, which is a contradiction.

We therefore have that $L$ is contained in the disjoint union of finitely many pairwise disjoint open intervals $J_{1}, \ldots, J_{m}$ the endpoints of which are not in $L$ and are joined by polygonal lines that miss $K$, except possibly for the two endpoints 
$a_{i}, b_{i}$ of each $J_{i}$. Let $U=\bigcup_{i=1, \ldots, m} J_{i}$; we have that $F=R \backslash U$ is the disjoint union of finitely many closed intervals (in particular, $F$ is compact) and misses $L$. So there is a finite union of rectangles $W \supset F$ so that if $R^{\prime}$ is a rational segment parallel to $R$ and $R_{1}^{\prime}=R^{\prime} \cap W$, then $R_{1}^{\prime} \cap K=\emptyset$. We now pick $R^{\prime}$ sufficiently close to $R$ so that $R_{1}^{\prime}$ intersects all the finitely many polygonal lines $P_{i}^{\prime}$. We therefore get that $R_{1}^{\prime} \cup \bigcup_{i=1, \ldots, m} P_{i}^{\prime} \backslash\left\{a_{i}, b_{i}\right\}$ is a connected set that intersects $U^{\prime}$ and $V^{\prime}$ and misses $K$, which is our final contradiction.

Remark. By the claim in proposition [3.1, it is clear that if $\tau$ is a topology on $[0,1]^{2}$ as in proposition 3.1 , then $\left([0,1]^{2}, \tau\right)$ has no countable open sets and that, if $x$ is one of the countably many exceptional points at which we have refined the topology, then $x$ has a local base consisting of open sets $U$ such that $U \backslash\{x\}$ is open in the usual Euclidean topology.

Before we proceed, we need the following set-theoretic lemma, a corollary of a theorem due to Bernstein, Kuratowski and Sierpiński, which, in turn, is a special case of a standard disjoint refinement lemma (see 2] for more information).

Lemma 3.2. Suppose that $B$ and $\left(D_{i}\right)_{i \in \omega}$ are infinite subsets of $\omega$. There exist infinite sets $B^{\prime} \subseteq B$ and $D_{i}^{\prime} \subseteq D_{i}$ such that $B^{\prime} \cap D_{i}^{\prime}=\emptyset$ for all $i \in \omega$.

Lemma 3.3. Suppose that $[0,1]^{2}$ has a separable metric topology $\tau$ as in proposition 3.1 and suppose that $U$ is a non-empty Euclidean open set in $[0,1]^{2}$ that is not dense. There exists a Cantor set (in the Euclidean topology) $K$ such that $S=B d(U) \supseteq K$ and a countable $D \subseteq K$ which is dense in $K$ with the Euclidean topology, dense in itself (in $\tau$ ) and such that, for all $d \in D$, there exists a rational segment $R_{d}$ such that $R_{d} \cap K=\{d\}$ and a clopen subset $C_{d}$ of $K$, and open squares $V_{n, d} \ni x_{n}^{d} \in D$ such that $d \in C_{d}$ and $C_{d} \backslash\{d\} \subseteq \bigcup_{n \in \omega} V_{n, d}, \mathcal{S}(d) \ni\left(\overline{V_{n, d}}\right)_{n \in \omega}$ and $\left(V_{n, d}\right)_{n \in \omega} \rightarrow d$ (in $\left.\tau\right)$.

Moreover, if for all $d \in D$ we have already been given countably many sequences converging to $d$, then we may arrange that a subsequence of each of these sequences is disjoint from $K$.

Let $D=\left\{d_{n}: n \in \omega\right\}$ and let $\left(x_{n}\right)_{n \in \omega}$ be an enumeration of the endpoints of the open intervals removed during the standard construction of $2^{\omega}$.

There also exists a homeomorphism $\phi: K \longrightarrow 2^{\omega}$ with respect to the Euclidean topologies of $K$ and $2^{\omega}$ such that $\phi\left(d_{n}\right)=x_{n}$ so that $\left(\phi^{-1}\left(x_{m}^{\prime}\right)\right)_{m \in \omega}$ and $\left(\phi^{-1}\left(x_{m}^{\prime \prime}\right)\right)_{m \in \omega}$ converge to $\phi^{-1}(x)$ in $\tau$ (where the sequences $\left(x_{m}^{\prime}\right)_{m \in \omega},\left(x_{m}^{\prime \prime}\right)_{m \in \omega}$ are as in lemma 2.2).

Proof. We have that $[0,1]^{2} \backslash S=U \cup V$, where $V=\bar{U}^{c}$. Since $U$ is non-empty and not dense, we may pick $p, q \in Q^{2}$ such that $p \in U, q \in V$ and let $R$ denote the rational segment joining $p, q$. We observe that there are squares $U^{\prime}, V^{\prime}$ as in proposition 3.1, and, using the hypotheses on $\left([0,1]^{2}, \tau\right)$, there exist $x \in R$, a sequence $x_{n} \rightarrow x$ and a sequence of rational segments $\left(R_{n}\right)_{n \in \omega}$ such that $x_{n} \in R_{n}$, $x_{n} \notin R, x_{n} \in M^{\prime} \cap S$. In the case that $x$ is one of the countably many exceptional points where we have refined the topology, we will have that, for all $n \in \omega, x_{n} \notin$ $\bigcup_{i \in \omega} B_{i, x}^{\alpha}$ and so that $x_{n} \rightarrow x$ in $\tau$.

Suppose now that we have countably many sequences converging to $x$. By an application of lemma 3.2 we may assume that the union of these sequences is disjoint from a subsequence of $x_{n}$, which we will suppose is still $x_{n}$. Without loss of generality, we may also assume that the union $\left(s_{n}\right)_{n \in \omega}$ of these sequences converges to $x$. Since $\left(B_{n, x}^{\alpha}\right)_{n \in \omega}$ converges to $x$ we may apply proposition 3.1 to pick open 
squares $\left(V_{n}\right)_{n \in \omega}$ which contain $x_{n}$, miss $R \cup\left\{s_{n}: n \in \omega\right\} \cup \bigcup_{n \in \omega} B_{n, x}^{\alpha}$ and so that $\left(\overline{V_{n}}\right)_{n \in \omega} \in \mathcal{S}(x)$. (We note here that if we haven't refined the topology at $x$, the proof is simpler, since we do not need to refer to the sequence $\left.\left(B_{n, x}^{\alpha}\right)_{n \in \omega}\right)$. So we may assume that $\operatorname{diam}\left(V_{n}\right)<\frac{1}{2}$ and we observe that each $\overline{V_{n}}$ is clopen in $\bigcup_{n \in \omega} \overline{V_{n}} \cup\{x\}=K_{0}$, hence $x$ has a clopen base in $K_{0}$ and, if we pick $d_{n} \in V_{n}$, $d_{n} \rightarrow x$ in $\tau$ (so in the usual topology as well).

We observe that since $x_{n} \in S$, each $V_{n}$ contains analogues of $U^{\prime}, V^{\prime}$ (as in the statement of proposition [3.1). So we can iterate this process to get that for all $n \in \omega$ and for each function $\sigma: n \longrightarrow \omega$ we can pick points $x_{\sigma} \in S$, open squares $V_{\sigma}$ and rational segments $R_{\sigma}$ so that $V_{\emptyset}=[0,1]^{2}, R_{\emptyset}=R, x_{\emptyset}=x$ and the following hold:

(i) $x_{\sigma} \in V_{\sigma}$ and $x_{\sigma} \in R_{\sigma}$,

(ii) if $\rho$ is an extension of $\sigma, \overline{V_{\rho}} \subseteq V_{\sigma}$,

(iii) $\left(\overline{V_{\sigma \frown n}}\right)_{n \in \omega} \in \mathcal{S}\left(x_{\sigma}\right)$ and if we have countably many sequences converging to $x_{\sigma}$, the open squares $V_{\sigma \sim n}$ converge to $x_{\sigma}$ in $\tau$ and miss a sequence which contains a subsequence of each of these sequences and the rational segment $R_{\sigma}$.

(iv) for fixed $\sigma$ with $\operatorname{dom}(\sigma)=m, \operatorname{diam}\left(V_{\sigma}\right)<\frac{1}{2^{m}}$.

Now let $K_{m+1}=\left\{x_{\sigma}: \operatorname{dom}(\sigma) \leq m\right\} \cup \bigcup\left\{\overline{V_{\sigma \sim n}}: n \in \omega, \sigma: m \longrightarrow \omega\right\}$. We have that $\left(K_{m}\right)_{m \geq 0}$ is a decreasing sequence of compact metric spaces in the usual topology (use (i), (ii) and (iii)) so their intersection $K$ is compact metric. It is also clear that each point $x_{\sigma}$ has a clopen base in $K_{d o m(\sigma)}$ (so also in $K$ ) and, using (iii), we get that $D=\left\{x_{\sigma}: \sigma \in \omega^{m}, m \in \omega\right\}$ is a countable dense in itself (in $\tau$ ) subset of $K$. Furthermore, for each $d=x_{\sigma} \in D$ we have that there exists a rational segment $R_{d}=R_{\sigma}$ with $R_{d} \cap K=\{d\}$. If we have countably many sequences converging to $d$ there is a sequence that contains a subsequence of each of these countably many sequences and is disjoint from $K$ (by (iii) again).

Finally, we use (iv) to conclude that $K \backslash D$ consists of the points that are the intersections of the closures of open sets in a branch of our tree of open sets, so we get that each of these $\mathfrak{c}$ many points (use (ii) again) is a limit point of $D$ (i.e. $K=\bar{D})$ and that it has a clopen local base. We now observe that $K$ is perfect ( $D$ is a dense in itself dense subset of $K$ ), compact, 0-dimensional metric space, hence $K$ is homeomorphic to the Cantor set and $D \subseteq S$ yields that $K \subseteq S$.

To finish the proof, just observe that if $d=x_{\sigma} \in D$, then $C_{d}=V_{\sigma} \cap K$ is the clopen set required in the statement of the lemma and that, if $d=x_{\sigma}$, then $x_{n}^{d}=x_{\sigma^{\wedge} n}$ and $V_{n, d}=V_{\sigma^{\wedge} n}$. Finally, any homeomorphism $\phi$ with $\phi(D)=\left\{x_{n}\right.$ : $n \in \omega\}$ will do, since, if $\phi^{-1}(x)=d=x_{\sigma}$, then the sequences $\left(\phi^{-1}\left(x_{m}^{\prime}\right)\right)_{m \in \omega}$, $\left(\phi^{-1}\left(x_{m}^{\prime \prime}\right)\right)_{m \in \omega}$ will eventually belong to $C_{d}$ and so $C_{d} \backslash\{d\} \subseteq \bigcup_{n \in \omega} V_{n, d}$ and $\left(V_{\sigma \frown n}\right)_{n \in \omega}$ converges to $x_{\sigma}$ in $\tau$.

For each $x \in[0,1]^{2} \backslash Q^{2}$ that belongs to a rational segment, let $S_{x}$ be the unique segment that goes through $x$ and has endpoints on the the unit square $[0,1]^{2}$ (this exists since the intersection of two non-parallel rational segments is a point in $Q^{2}$ ). For each $x \in Q^{2}$, let $S_{x}=\{x\}$. Let $\mathcal{N}=\mathcal{B} \cup \mathcal{Q}$ be the (countable) network for $[0,1]^{2}$, where $\mathcal{B}$ is a countable base for $[0,1]^{2}$ and $\mathcal{Q}$ is the family of rational segments in $[0,1]^{2}$.

We now prove a lemma that will provide the method of construction at a successor stage. 
Lemma 3.4. Suppose that $X \subseteq[0,1]^{2}$ contains all rational segments, and, whenever $C$ is a copy of the Cantor set in $[0,1]^{2}, C \backslash X$ is countable.

Suppose that $\tau$ is a stronger separable metric topology on $[0,1]^{2}$ as in proposition [3.1 such that, if $x \in[0,1]^{2}$ is one of the countably many exceptional points where we have refined the topology and $x \in U, U$ is open in $\tau$ and $R_{x}$ is a rational segment such that $x \in R_{x} \subseteq S_{x}$, then there is a rational segment $R \subseteq R_{x}$ such that $x \in R \subseteq U$ (so, in particular, we assume $\mathcal{N}$ is a (countable) network for $\left([0,1]^{2}, \tau\right)$ ).

Suppose $K$ is a Cantor subset of $[0,1]^{2}$ such that $(K \cap X, \tau \uparrow K \cap X)$ is 0 dimensional and suppose $D=\left\{d_{n}: n \in \omega\right\}$ is a countable dense subset of $K$. Let $\left(x_{n}\right)_{n \in \omega}$ be an enumeration of the endpoints of the open intervals removed during the standard construction of $2^{\omega}$. Suppose that the following hold:

(1) For each $d \in D$ there exists a rational segment $R_{d}$ with $R_{d} \cap K=\{d\}$ (so, in particular, $D \subseteq X)$ and a sequence $\left(y_{n}^{d}\right)_{n \in \omega}$ converging to $d$ in $\tau$ that is disjoint from $K$.

(2) For each $d \in D$ there exist a clopen subset $C_{d}$ of $K$ and open squares $V_{n, d} \ni$ $x_{n}^{d} \in D$ such that $d \in C_{d}$ and $C_{d} \backslash\{d\} \subseteq \bigcup_{n \in \omega} V_{n, d}, \mathcal{S}(d) \ni\left(\overline{V_{n, d}}\right)_{n \in \omega}$ and $\left(V_{n, d}\right)_{n \in \omega} \rightarrow d($ in $\tau)$.

(3) There exists $\phi: K \longrightarrow 2^{\omega}$ (a homeomorphism with respect to the usual topologies of $K$ and $\left.2^{\omega}\right)$ such that $\phi\left(d_{n}\right)=x_{n}$ and $\left(\phi^{-1}\left(x_{m_{i}}^{\prime}\right)\right)_{i \in \omega},\left(\phi^{-1}\left(x_{m_{j}}^{\prime \prime}\right)\right)_{j \in \omega}$ converge to $\phi^{-1}(x)$ in $\tau$ (where the sequences $\left(x_{m}^{\prime}\right)_{m \in \omega},\left(x_{m}^{\prime \prime}\right)_{m \in \omega}$ are as in lemma 2.2).

Let $Z$ the space obtained by resolving $\left([0,1]^{2}, \tau\right)$ at each point $d$ of $D$ into $[-1,1]$ (all the other $Y_{x}$ 's are $\left.=\left\{c_{x}\right\}\right)$ so that $f_{d}\left(\left\{y_{n}^{d}: n \in \omega\right\} \cup R_{d} \backslash\{d\}\right)=x_{d}=f(\phi(d)$ ) (where $f$ is the Kuratowski function).

Let

$$
\begin{aligned}
Z^{\prime} & =\bigcup_{d \in D}\left\{\left(d, x_{d}\right)\right\} \cup \bigcup_{x \in X \backslash D}\left\{\left(x, c_{x}\right)\right\} \subseteq Z^{\prime \prime} \\
& =\bigcup_{d \in D}\left\{\left(d, x_{d}\right)\right\} \cup \bigcup_{x \notin D}\left\{\left(x, c_{x}\right)\right\} \subseteq Z
\end{aligned}
$$

and let $\pi: Z \longrightarrow[0,1]^{2}$ be the continuous projection.

Then

(i) $\operatorname{dim} \pi^{-1}(K) \cap Z^{\prime}=1$,

(ii) $Z^{\prime}$ (even $Z$ ) is separable metric,

(iii) $\left\{\pi^{-1}(N) \cap Z^{\prime \prime}: N \in \mathcal{N}\right\}$ is a countable network for $Z^{\prime \prime}$,

(iv) $\left(y_{n}^{d}, c_{y_{n}^{d}}\right)_{n \in \omega}$ converges to $\left(d, x_{d}\right)$, and

(v) if $\operatorname{dim}(X, \tau \uparrow X)=n \geq 1$, then $\operatorname{dim} Z^{\prime} \leq n$; if $n=1$, then $\operatorname{dim} Z^{\prime}=1$.

Let $\tau^{\prime}$ be the quotient topology induced by $\pi \uparrow Z^{\prime \prime}$ on $[0,1]^{2}$.

Then, moreover, $\left(K \cap X, \tau^{\prime} \uparrow K \cap X\right)$ is 1-dimensional, $\left([0,1]^{2}, \tau^{\prime}\right)$ is a separable metric space and $\left(y_{n}^{d}\right)_{n \in \omega}$ converges to $d$ in $\tau^{\prime}$.

Furthermore, if $\operatorname{dim}(X, \tau \uparrow X)=n \geq 1$, then $\operatorname{dim}\left(X, \tau^{\prime} \uparrow X\right) \leq n$. In particular, if $n=1$, then $\operatorname{dim} Z^{\prime}=1$.

Moreover, suppose that $x \in[0,1]^{2}$ is one of the countably many points at which the new topology is finer than the Euclidean one. Then $x$ belongs to a rational segment, and, whenever $U$ is a basic open neighborhood of $x$ in the new topology,

(a) $U \backslash\{x\}=V$ is open in the Euclidean topology.

(b) $U$ contains an open square minus the union of a tail of a sequence $\left(B_{i, x}^{\alpha}\right)_{i \in \omega} \in$ $\mathcal{S}(x)$ for some fixed $\alpha<\omega_{1}$. 
(c) if $R_{x}$ is a rational segment such that $x \in R_{x} \subseteq S_{x}$, then there exists a rational segment $R \subseteq R_{x}$ such that $x \in R \subseteq U$.

Proof. We first define the functions $f_{d}$ for $d \in D$. Fix a $C_{d}$ as in (2); with $\phi$ as in (3), define $f_{d}\left\lceil C_{d} \backslash\{d\}=g_{\phi(d)} \circ \phi\left\lceil C_{d} \backslash\{d\}\right.\right.$ where $g_{\phi(d)}$ is as in lemma 2.3 and define $f_{d}\left\lceil\left(\bigcup_{n \in \omega} V_{n, d}\right)^{c} \cup\left\{y_{n}^{d}: n \in \omega\right\} \cup R_{d} \backslash\{d\}\right.$ to be equal to the constant $x_{d}=f(\phi(d))$, where $f$ is the Kuratowski function.

Now

$$
\begin{gathered}
\left(\left(\bigcup_{n \in \omega} V_{n, d}\right)^{c} \cup \overline{\left\{y_{n}^{d}: n \in \omega\right\}} \cup R_{d}\right) \cap C_{d}=\{d\}, \\
f_{d} \uparrow\left(\bigcup_{n \in \omega} V_{n, d}\right)^{c} \cup \overline{\left\{y_{n}^{d}: n \in \omega\right\}} \cup R_{d} \cup C_{d} \backslash\{d\} \\
=f_{d} \uparrow\left(\bigcup_{n \in \omega} V_{n, d}\right)^{c} \cup\left\{y_{n}^{d}: n \in \omega\right\} \cup R_{d} \cup C_{d} \backslash\{d\}
\end{gathered}
$$

is well-defined and continuous by the pasting lemma). So, since $\left(\bigcup_{n \in \omega} V_{n, d}\right)^{c} \cup$ $\overline{\left\{y_{n}^{d}: n \in \omega\right\}} \cup R_{d} \cup C_{d} \backslash\{d\}$ is closed in $[0,1]^{2} \backslash\{d\}$, we deduce that $f_{d}\left\lceil\left(\bigcup_{n \in \omega} V_{n, d}\right)^{c} \cup\right.$ $\overline{\left\{y_{n}^{d}: n \in \omega\right\}} \cup R_{d} \cup C_{d} \backslash\{d\}$ can be extended to a continuous function on $[0,1]^{2} \backslash\{d\}$ by Tietze's extension theorem.

Finally, if $x \in K \backslash D$ let $c_{x}=f(\phi(x))$ and if $x \notin K$, let $c_{x}=0$. (Note that the $f_{d}$ 's are continuous even when $[0,1]^{2}$ has the usual Euclidean topology.)

So $Z$ is a separable metric space (by corollary [1.3), and $Z^{\prime}$ is a separable metric space and (ii) is proved.

We observe that $\pi^{-1}(K) \cap Z^{\prime}=\{(x, f(\phi(x)): x \in K \cap X\} \subseteq\{(x, f(\phi(x)): x \in$ $K\}=L$. We will show that $L$ is homeomorphic to the graph of the Kuratowski function if we suppose that $2^{\omega}$ gets the topology $\rho$ that makes $\phi:\left(K, \tau\lceil K) \longrightarrow 2^{\omega}\right.$ a homeomorphism. In particular, this will imply that

$$
\operatorname{dim} \pi^{-1}(K) \cap Z^{\prime} \leq \operatorname{dim}((K \cap X, \tau \uparrow K \cap X) \times[-1,1])=1
$$

since $(K \cap X, \tau \uparrow K \cap X)$ is 0-dimensional.

Indeed, the map $i$ defined by $i(x, f(\phi(x)))=(\phi(x), f(\phi(x)))$ is clearly 1-1 and onto and we will show it is a homeomorphism.

Suppose that $\left(x_{n}, f\left(\phi\left(x_{n}\right)\right)\right) \rightarrow(x, f(\phi(x)))$ in the resolution topology. We get that $x_{n} \rightarrow x$ and that $\phi\left(x_{n}\right) \rightarrow \phi(x)$ (both sequences converge in the stronger, so in the usual topologies as well), so if $\phi(x)$ is a point of continuity of $f$, we are done.

If this is not the case, we get that $x \in D$ and that $g_{\phi(x)}\left(\phi\left(x_{n}\right)\right) \rightarrow f(\phi(x))$. Indeed, recall that a basic open set containing $(x, f(\phi(x)))$ is $\{(x, f(\phi(x)))\} \cup$ $\bigcup\left\{\left(x^{\prime}, f\left(\phi\left(x^{\prime}\right)\right)\right): x^{\prime} \in U \cap\left(g_{\phi(x)} \circ \phi\lceil K \backslash\{x\})^{-1}(W)\right\}\right.$ (where $U \ni x$ and $U$ is open, $W \ni f(\phi(x))$ and $W$ is open) and apply that to get that for sufficiently large $n,\left(g_{\phi(x)} \circ \phi\lceil K \backslash\{x\})\left(x_{n}\right) \in W\right.$. Therefore $g_{\phi(x)}\left(\phi\left(x_{n}\right)\right) \rightarrow f(\phi(x))$ and the construction of $g_{\phi(x)}$ yields that $f\left(\phi\left(x_{n}\right)\right) \rightarrow f(\phi(x))$. This shows that $i$ is continuous.

Conversely, suppose that $\phi\left(x_{n}\right) \rightarrow \phi(x)$ and $f\left(\phi\left(x_{n}\right)\right) \rightarrow f(\phi(x))$. Thus $\phi\left(x_{n}\right) \rightarrow$ $\phi(x)$ in $\rho$ and the construction of $g_{x}$ yields that $g_{\phi(x)}\left(\phi\left(x_{n}\right)\right) \rightarrow f(\phi(x))$ when $\phi(x)$ is not a point of continuity of $f$. So again $\left(x_{n}, f\left(\phi\left(x_{n}\right)\right)\right) \rightarrow(x, f(\phi(x)))$ in the resolution topology. If $\phi(x)$ is a point of continuity, then $f_{x}$ is a constant $\left(=c_{x}\right)$ and the proof is trivial. This shows that $i^{-1}$ is continuous.

We therefore get that $\pi^{-1}(K) \cap Z^{\prime}$ is homeomorphic to $G r(f) \backslash H$, where $H=\left\{\left(\phi(x), f(\phi(x)): x \in K \cap X^{c}\right\}\right.$ is countable and $p_{1}(H) \cap\left\{x_{n}: n \in \omega\right\}=\emptyset$. 
We now check that a closed dense in itself subset $S$ of $(K, \tau \uparrow K)$ is still uncountable. Certainly $S=S_{\tau}^{\prime}=\bar{S}^{\tau}$. Also $\bar{S}=S \cup S^{\prime}=S_{\tau}^{\prime} \cup S^{\prime} \subseteq S^{\prime} \subseteq \bar{S}^{\prime}$ and $\bar{S}$ is closed dense in itself in the Euclidean topology, and thus uncountable. Thus $S$ is uncountable since $\left|\bar{S} \backslash \bar{S}^{\tau}\right| \leq \omega$ and we only refined the topology at countably many points.

By an application of proposition [2.1] since the sequences $\left(x_{m_{i}}^{\prime}\right)_{i \in \omega},\left(x_{m_{j}}^{\prime \prime}\right)_{j \in \omega}$ converge to $x$ in $\rho$, we get that $\operatorname{dim} \pi^{-1}(K) \cap Z^{\prime} \geq 1$ and (i) follows.

We now prove (iii). If $f_{x}$ is a constant map, a neighbourhood of $\left(x, c_{x}\right)$ in $Z^{\prime \prime}$ is of the form $V=\left\{\left(x, c_{x}\right)\right\} \cup \bigcup\left\{\left\{x^{\prime}\right\} \times Y_{x^{\prime}}: x^{\prime} \in U \backslash\{x\}\right\} \cap Z^{\prime \prime}$; let $x \in N \subseteq U$, where $N \in \mathcal{N}$. Now $\left(x, c_{x}\right) \in \pi^{-1}(N) \cap Z^{\prime \prime} \subseteq V$. Suppose that $(d, f(\phi(d))) \in$ $Z^{\prime \prime} \cap\left(\{d\} \times W \cup \bigcup\left\{\{x\} \times Y_{x}: x \in U \cap f_{d}^{-1}(W)\right\}\right)=V$ and observe that if $d \in U$ and $U$ is open in $\tau$, we have that there exists a rational segment $R \subseteq R_{d}$ (where $R_{d}$ is as in (1)) with $d \in R \subseteq U$. Since $f_{d}(R \backslash\{d\}) \subseteq f_{d}\left(R_{d} \backslash\{d\}\right)=x_{d}=f(\phi(d)) \in W$ and $R \subseteq U$, we deduce that $(d, f(\phi(d))) \in \pi^{-1}(R) \cap Z^{\prime \prime} \subseteq V$.

For (iv), we just need to apply the definition of the resolution topology.

Finally, we prove $(\mathrm{v})$. Since $\pi \uparrow \pi^{-1}(X \backslash D)$ is a homeomorphism, $\pi^{-1}(X \backslash K)$ is an open $F_{\sigma}$ set of dimension $\leq n$, so $\operatorname{dim} Z^{\prime} \leq n$ by an application of the countable sum theorem and (i). The case $n=1$ is trivial.

Now $f_{d} \uparrow\left(\bigcup_{n \in \omega} V_{n, d}\right)^{c}=x_{d}=f(\phi(d))$ and the resolution functions are continuous in the Euclidean topology. So, for any basic $\tau^{\prime}$-open set $U$ containing $d$, there exists some $\tau$-open $V$ such that $U \supseteq V \backslash \bigcup_{n \in \omega} \overline{V_{n, d}}$ by (II) in proposition 3.1 Using proposition 3.1 we get the last paragraph of this lemma.

It is indeed possible to have a subset $X$ of $[0,1]^{2}$ as in lemma 3.4 so that $X$ is $\mathfrak{c}$-dense in $[0,1]^{2}$ (i.e. $|X \cap V|=\mathfrak{c}$ for all $V \subseteq[0,1]^{2}, V$ open and non-empty) and $[0,1]^{2} \backslash X$ is dense in $[0,1]^{2}$. This is established in the following

Lemma 3.5. There exists $Y \subseteq[0,1]^{2}$ such that $Y$ contains all rational segments and $[0,1]^{2} \backslash Y$ is a countable dense subset of $[0,1]^{2}$. We conclude that if $C$ is a copy of the Cantor set in $[0,1]^{2}, C \cap Y^{c}$ is countable (therefore $Y$ is c-dense in $[0,1]^{2}$ ) and that $\operatorname{dim} Y=1$.

Proof. The complement of a rational segment is open and dense in $[0,1]^{2}$, therefore an application of the Baire category theorem yields that $A=[0,1]^{2} \backslash \bigcup \mathcal{Q}$ (where $\mathcal{Q}$ is the family of rational segments) is dense in $[0,1]^{2}$. (We note here that a fancy way to see this is that $\bigcup \mathcal{Q}$ is 1 -dimensional (apply the countable sum theorem), hence it has no interior in $[0,1]^{2}$ ).

Let $D$ be a countable dense subset of $A$ (therefore, $D$ is dense in $[0,1]^{2}$ as well) and let $Y=[0,1]^{2} \backslash D$. We only verify that $\operatorname{dim} Y=i n d Y=1$, as everything else is immediate. Indeed, $Y$ has no interior in $[0,1]^{2}$, therefore ind $Y \leq 1$ (see [3], Corollary 1.8.11.) and we actually have that ind $Y=1$ as $Y$ contains all rational segments.

Before we construct our space $X$, we will state and prove the following

Lemma 3.6. Suppose that $\left(\tau_{\alpha}\right)_{\alpha<\omega_{1}}$ is an increasing continuous sequence of topologies on a set $X$ (i.e. if $\alpha$ is a limit ordinal, $\left.\sup \left\{\tau_{\beta}: \beta<\alpha\right\}=\tau_{\alpha}\right)$ so that $\left(X, \tau_{\alpha}\right)$ is a separable metric space for all $\alpha<\omega_{1}$ and $\mathcal{N}=\left\{N_{i}: i \in \omega\right\}$ is a countable network for $(X, \tau)$, where $\tau=\sup \left\{\tau_{\alpha}: \alpha<\omega_{1}\right\}$.

We then have that the following are true: 
(i) If $V \in \tau$, there exists $\alpha<\omega_{1}$ such that $V \in \tau_{\alpha}$ and, therefore, if there exists $\alpha<\omega_{1}$ such that for all $\beta>\alpha, \operatorname{dim}\left(X, \tau_{\beta}\right) \leq n$, then $\operatorname{dim}(X, \tau) \leq n$.

(ii) If $S \subseteq X$, there exists $\alpha<\omega_{1}$ such that $\bar{S}^{\tau}=\bar{S}^{\tau_{\beta}}$ for all $\beta>\alpha$, and therefore, there exists $\gamma<\omega_{1}$ such that $B d_{\tau}(S)=B d_{\tau_{\beta}}(S)$ for all $\beta>\gamma$.

(iii) If ind $(X, \tau)=0$, we have that there exists $\alpha<\omega_{1}$ such that ind $\left(X, \tau_{\alpha}\right)=0$.

Proof. (i) We observe that a basic open set $V$ in $\tau$ will be open in some intermediate topology $\left(V=G_{1} \cap \ldots \cap G_{k}\right.$, where $k \in \omega$ and $G_{i} \in \tau_{\alpha_{i}}, i=1, \ldots, k$; therefore if $\alpha>\alpha_{i}$ for $i=1, \ldots, k$ we get that $\left.V \in \tau_{\alpha}\right)$. We use that $X$ has a countable network to get that $X$ is hereditarily Lindelöf, so we may have that an open subset $V$ of $X$ will be the countable union of basic open sets and the argument above yields that $V \in \tau_{\alpha}$ for some $\alpha<\omega_{1}$ (use that $\omega_{1}$ is regular).

This fact shows that $\operatorname{dim} X \leq n$; indeed, let $\left(V_{i}\right)_{i=1, \ldots, k}$ be a finite open cover of $X$, let $\alpha<\omega_{1}$ be such that $V_{i} \in \tau_{\alpha}$ for all $i=1, \ldots, k$ and $\operatorname{dim} X_{\alpha} \leq n$ to get an open refinement of order $\leq n$ in $\tau_{\alpha}$ which is the desired refinement.

(ii) Let $S$ be as above and let $\bar{S}^{\tau_{\alpha}}$ be the closure of $S$ in $\tau_{\alpha}$; these sets are closed in $\tau$ and form a decreasing family of size $\omega_{1}$, so, since $(X, \tau)$ is hereditarily Lindelöf, it stabilizes, i.e. $\bar{S}^{\tau_{\beta}}=\bar{S}^{\tau_{\alpha}} \forall \beta>\alpha$ for some $\alpha<\omega_{1}$. We also observe that $\bar{S}^{\tau}=\bigcap_{\beta<\omega_{1}} \bar{S}^{\tau_{\beta}}$. Indeed, let $x \notin \bar{S}^{\tau}$ and let $x \in G, G$ basic open with $G \cap S=\emptyset$; get $\beta<\omega_{1}$ such that $G \in \tau_{\beta}$ and see that $x \notin \bar{S}^{\tau_{\beta}}$. These two observations finish the proof of the first part of (ii) and the second part follows from this and (i).

(iii) Let $\alpha_{0}=0$; we will construct an increasing sequence of ordinals $\left(\alpha_{n}\right)_{n \in \omega}$ such that, if $N \in \mathcal{N}, N \subseteq U$, and $U$ is a basic open set in $\tau_{\alpha_{n}}$, there exists a clopen (in $\tau$ ) set $H$ such that $N \subseteq H \subseteq U$, and $H, H^{c} \in \tau_{\alpha_{n+1}}$, i.e. $H$ is clopen in $\tau_{\alpha_{n+1}}$.

Indeed, this is possible, since $\left(X, \tau_{\alpha}\right)$ is a separable metric space $\alpha<\omega_{1}$ and $\mathcal{N}$ is countable; use (i), that $\omega_{1}$ is regular and that $\operatorname{Ind}(X, \tau)=0$. (Recall that $(X, \tau)$ is Lindelöf).

We let $\alpha=\sup \left\{\alpha_{n}: n \in \omega\right\}$ and we claim that this $\alpha$ works. Indeed, let $x \in U$, where $U$ is basic open in $\tau_{\alpha}$. We may assume that $U$ is basic open in $\tau_{\alpha_{n}}$ for some $n \in \omega$, so there exists a clopen (in $\tau_{\alpha_{n+1}} \subseteq \tau_{\alpha}$ ) set $H$ and $N \in \mathcal{N}$ such that $x \in N \subseteq H \subseteq U$ and the proof is complete.

Theorem 3.7 $(\mathrm{CH})$. There exists a regular space $X$ with a countable network such that $\operatorname{dim} X=1$ and ind $X=$ Ind $X \geq 2$.

Proof. Let $Y$ be as in lemma 3.5. So ind $Y=1, Y$ contains all rational segments and, whenever $C$ is a copy of the Cantor set in $[0,1]^{2}, C \backslash Y^{c}$ is countable. Thus $Y$ is $\mathfrak{c}$-dense in $[0,1]^{2}$.

Using $\mathrm{CH}$, let $\left(B d\left(U_{\alpha}\right)\right)_{\alpha<\omega_{1}}$ be a list of all boundaries of all non-empty open subsets of $[0,1]^{2}$ that are not dense so that each $B d\left(U_{\alpha}\right)$ is listed stationarily often (we will need stationary sets rather than unbounded sets in the proof of claim 2 below).

For each $\alpha<\omega_{1}$, we will construct a topology $\tau_{\alpha}$ on $[0,1]^{2}$, two sets $K_{\alpha}, D_{\alpha} \subseteq$ $[0,1]^{2}$ and a function $\phi_{\alpha}: K_{\alpha} \longrightarrow 2^{\omega}$ such that the following will hold:

(i) $\forall \alpha<\beta<\omega_{1}, \tau_{\alpha} \subseteq \tau_{\beta}$.

(ii) $\forall \alpha<\omega_{1}, X_{\alpha}=\left(Y, \tau_{\alpha}\right)$ is a separable metric space.

(iii) In each topology $\tau_{\alpha}$, all but countably many points of $[0,1]^{2}$ will have a local base of Euclidean open sets; and if $x$ is one of these countably many points, $x$ belongs to a rational segment and has a local base so that any element of the base 
contains an open square minus the union of a tail of some sequence $\left(B_{i, x}^{\alpha}\right)_{i \in \omega} \in \mathcal{S}(x)$ (recall the definition in proposition 3.1).

(iv) In each topology $\tau_{\alpha}$, if $x \in[0,1]^{2}$ is one of the countably many points at which we have refined the topology and $x \in U, U$ is open in $\tau_{\alpha}$ and $R_{x}$ is a rational segment such that $x \in R_{x} \subseteq S_{x}$ ( $S_{x}$ is defined in the paragraph before the statement of lemma 3.4), we have that $x \in R \subseteq U$ for some rational segment $R \subseteq R_{x}$. (In particular, for every $\alpha<\omega_{1}, \mathcal{N}$ is a countable network for $\left([0,1]^{2}, \tau_{\alpha}\right)$ (recall the definition just before lemma 3.4).

(v) for all $\alpha<\omega_{1}, \operatorname{dim}\left(Y, \tau_{\alpha} \uparrow Y\right)=1$.

(vi) $K_{\alpha}$ is a Cantor set (in the Euclidean topology) that is contained in $B d\left(U_{\alpha}\right)$ whenever $\left(B d\left(U_{\alpha}\right) \cap Y, \tau_{\alpha} \uparrow B d\left(U_{\alpha}\right) \cap Y\right)$ is 0-dimensional; otherwise, $K_{\alpha}=\emptyset$.

(vii) $D_{\alpha} \subseteq Y$ is a countable dense subset of $K_{\alpha}$.

(viii) $\phi_{\alpha}: K_{\alpha} \longrightarrow 2^{\omega}$ is a homeomorphism (with respect to the usual topologies of $K_{\alpha}$ and $\left.2^{\omega}\right)$ such that $\phi_{\alpha}\left(d_{n}^{\alpha}\right)=x_{n}$ where $D_{\alpha}=\left\{d_{n}^{\alpha}: n \in \omega\right\}$ and $\left(x_{n}\right)_{n \in \omega}$ is an enumeration of the endpoints of the open intervals removed during the standard construction of $2^{\omega}$, whenever $K_{\alpha} \neq \emptyset$; otherwise, $\phi_{\alpha}=\emptyset$.

(ix) If $\beta<\alpha$, both sequences $\left(\phi_{\beta}^{-1}\left(x_{m}^{\prime}\right)\right)_{m \in \omega},\left(\phi_{\beta}^{-1}\left(x_{m}^{\prime \prime}\right)\right)_{m \in \omega}$ have a subsequence that converges to $\phi_{\beta}^{-1}(x)$ in $\tau_{\alpha}$ (recall the definition in lemma 2.2).

Now we describe the inductive construction of the $\tau_{\alpha}, K_{\alpha}, D_{\alpha}$ and $\phi_{\alpha}$.

Suppose that we have defined $\left\{\tau_{\gamma}, K_{\xi}, D_{\xi}, \phi_{\xi}: \xi<\beta, \gamma \leq \beta\right\}$ for each $\beta<\alpha$. If $\alpha$ is a limit ordinal, then we only define $\tau_{\alpha}=\sup \left\{\tau_{\beta}: \beta<\alpha\right\}$ (this implies $X_{\alpha}=\lim \left\{X_{\beta}: \beta<\alpha\right\}$; see section 11).

Now suppose $\alpha=\beta+1$. We will define $\tau_{\alpha}, K_{\beta}, D_{\beta}$ and $\phi_{\beta}$.

If $\left(B d\left(U_{\beta}\right) \cap Y, \tau_{\beta} \uparrow B d\left(U_{\beta}\right) \cap Y\right)$ is not 0-dimensional, then we let $\tau_{\alpha}=\tau_{\beta}$ and $K_{\beta}=D_{\beta}=\phi_{\beta}=\emptyset$.

If $\left(B d\left(U_{\beta}\right) \cap Y, \tau_{\beta} \uparrow B d\left(U_{\beta}\right) \cap Y\right)$ is 0-dimensional, then we apply lemma 3.3 (with $U=U_{\beta}$ and $\tau=\tau_{\beta}$ ) to get $K_{\beta}, D_{\beta}$ and $\phi_{\beta}$ so that:

(0) $K_{\beta}$ is a Cantor set (in the Euclidean topology) such that $K_{\beta} \subseteq B d\left(U_{\beta}\right)$.

(1) $K_{\beta}$ has a dense subset $D_{\beta}$ such that, if $d \in D_{\beta}$, then there exists a rational segment $R_{d}$ such that $d \in R_{d}, R_{d} \cap K_{\beta}=\{d\}$ and if $d \in D_{\gamma}$ for some $\gamma<$ $\beta$, and $\phi_{\gamma}(d)=x$, then the union $\left(y_{n}^{d}\right)_{n \in \omega}$ of some subsequences $\left(\phi_{\gamma}^{-1}\left(x_{m_{i}}^{\prime}\right)\right)_{i \in \omega}$, $\left(\phi_{\gamma}^{-1}\left(x_{m_{j}}^{\prime \prime}\right)\right)_{j \in \omega}$ converge to $\phi_{\gamma}^{-1}(x)=d$ in $\tau_{\beta}$ and are disjoint from $K_{\beta}$.

(2) For all $d \in D_{\beta}$, there exist a clopen subset $C_{d}$ of $K_{\beta}$ and open squares $V_{n, d} \ni x_{n}^{d} \in D_{\beta}$ such that $d \in C_{d}, C_{d} \backslash\{d\} \subseteq \bigcup_{n \in \omega} V_{n, d}, \mathcal{S}(d) \ni\left(\overline{V_{n, d}}\right)_{n \in \omega}$ and $\left(V_{n, d}\right)_{n \in \omega} \rightarrow d\left(\right.$ in $\left.\tau_{\beta}\right)$.

(3) $\phi_{\beta}: K_{\beta} \longrightarrow 2^{\omega}$ is a homeomorphism with respect to the usual topologies of $K_{\beta}$ and $2^{\omega}$ and $\phi_{\beta}\left(d_{n}^{\beta}\right)=x_{n}$, where $D_{\beta}=\left\{d_{n}^{\beta}: n \in \omega\right\}$ and $\left(x_{n}\right)_{n \in \omega}$ is an enumeration of the endpoints of the open intervals removed during the standard construction of $2^{\omega}$, so that $\left(\phi_{\beta}^{-1}\left(x_{m}^{\prime}\right)\right)_{m \in \omega},\left(\phi_{\beta}^{-1}\left(x_{m}^{\prime \prime}\right)\right)_{m \in \omega}$ converge to $\phi_{\beta}^{-1}(x)$ in $\tau_{\beta}$.

Note that we get (1) by applying our hypothesis (ix) and the fact that we can "put together" countably many sequences in a first countable space (i.e. if $Z$ is a topological space, $z \in Z$ has countable character and we have countably many sequences converging to $z$, then there is a sequence that converges to $z$ which contains all but finitely many elements of each of these sequences).

We now show that conditions (i)-(ix) are valid.

It is obvious that (i) and (ii) are valid and our construction guarantees that (iii) is satisfied, since if $\alpha$ is a successor, (iii) follows by lemma 3.4 while if $\alpha$ is a limit 
ordinal, (iii) follows from the definition of $X_{\alpha}$ (or, equivalently, from the definition of $\tau_{\alpha}$ ) and part (II) of proposition 3.1. We also have that lemma 3.4 yields that (iv) is valid if $\alpha$ is a successor and if $\alpha$ is a limit ordinal, (iv) follows from the definition of $\tau_{\alpha}$. Conditions (vi), (vii) and (viii) follow by our construction.

Now we verify (ix). Our requirements (1) and (3) (in the construction of all previous Cantor sets) and lemma 3.4 guarantee that (ix) is true in the case of a successor ordinal $\alpha$. If $\alpha$ is limit, then finding the appropriate subsequences (as in (ix)) is equivalent to finding an infinite set all but finitely many elements of which are contained in all the infinite sets corresponding to the subsequences we have at the previous stages of our inductive construction. These infinite subsets of $\omega$ form a decreasing (mod finite) sequence, and so this can be done.

Now we verify (v). If $\alpha$ is a successor ordinal, we apply lemma 3.4 So suppose $\alpha$ is a limit ordinal. An application of a theorem of Nagami (see 3], theorem 1.13.4.) implies that $\operatorname{dim} X_{\alpha}=i n d X_{\alpha} \leq 1$. So we will be done with (v) by proving the following claim.

Claim 1: If $\beta<\alpha<\omega_{1}$ and $K_{\beta} \neq \emptyset$, then $\left(K_{\beta} \cap Y, \tau_{\alpha}\left\lceil K_{\beta} \cap Y\right)\right.$ is 1-dimensional at the points $d \in D_{\beta}$.

Proof of claim 1: We need to check that the hypotheses of proposition 2.1 are satisfied with $\tau=\tau_{\alpha}$. But $\tau_{\alpha}$ is a separable metric topology that is stronger than the usual one and we have refined the topology at only countably many points. In particular, this means that a closed dense in itself subset of $2^{\omega}$ (with the refined topology) is uncountable.

So now we need only use (ix).

Now we have checked that (i)-(ix) are true.

We make a further claim before continuing.

Claim 2: For all $\gamma<\omega_{1}$, there exists $\alpha>\gamma, \alpha<\omega_{1}$ such that $B d\left(U_{\alpha}\right)=B d\left(U_{\gamma}\right)$ and $\operatorname{dim}\left(B d\left(U_{\alpha}\right) \cap Y, \tau_{\beta}\left\lceil B d\left(U_{\alpha}\right) \cap Y\right)=1\right.$ for all $\beta>\alpha$.

Proof of claim 2: Let $B d\left(U_{\gamma}\right)$ be given and observe that the set of countable ordinals $\delta$ such that $\left(B d\left(U_{\gamma}\right) \cap Y, \tau_{\delta}\left\lceil B d\left(U_{\gamma}\right) \cap Y\right)\right.$ is 0-dimensional, is closed. If it is also unbounded, we get that (recall that we listed each $B d\left(U_{\alpha}\right)$ stationarily often) there exists $\alpha<\omega_{1}$ such that $B d\left(U_{\alpha}\right)=B d\left(U_{\gamma}\right)$ and $\left(B d\left(U_{\alpha}\right) \cap Y, \tau_{\alpha}\left\lceil B d\left(U_{\alpha}\right) \cap Y\right)\right.$ is 0-dimensional. But, in this case, we do refine the topology of $Y$ to get a Cantor subset $K$ of $B d\left(U_{\alpha}\right)$ and a dense subset $D$ of $K$ such that $K \cap Y$ remains 1dimensional at all points $d \in D$ in all later topologies.

Now let $X=\lim _{\longleftarrow}\left\{X_{\alpha}: \alpha<\omega_{1}\right\}$. We see that $X$ is regular, that the topology $\tau$ of $X$ is the supremum of all intermediate separable metric topologies and that $\{N \cap X: N \in \mathcal{N}\}$ is a countable network for $X$. Another application of lemma 3.6 yields that $\operatorname{dim} X=1$ (use (i) and (iii) of lemma 3.6) and that non-empty open subsets of $X$ are uncountable.

Finally, we will show that if $U$ is a non-empty open subset of $X$ that is not dense, ind $B d(U) \geq 1$, so ind $X \geq 2$. (We note here that we get another proof that $\operatorname{dim} X=1$, since if $\operatorname{dim} X=0$, then $\operatorname{Ind} X=i n d X=0$, which would be impossible).

Then let $U$ be a non-empty open subset of $X$ that is not dense and apply lemma 3.6 to get $\alpha<\omega_{1}$ such that $U$ is open in $\tau_{\alpha}$ and $B d_{\tau_{\alpha}}(U)=B d_{\tau}(U)$. Observe that $B d_{Y}(U)=B d_{\tau_{\alpha}}(U) \cup H$, where $H$ is a countable set, and that if $V=\operatorname{Int}_{Y}(U)$, $U=V \cup L$, where $L$ is a countable set. We conclude that $V \neq \emptyset$ and that if $V=W \cap Y$ where $W$ is an open subset of $[0,1]^{2}, W \neq \emptyset$ and $B d_{Y}(V)=\bar{V}^{Y} \backslash V=$ 
$\overline{W \cap Y}^{Y} \backslash W \cap Y=\left(Y\right.$ is dense in $\left.[0,1]^{2}\right) \bar{W} \cap Y \backslash W \cap Y=B d(W) \cap Y$.

We also have that $\bar{U}^{Y}=\bar{V}^{Y}$; for this, it suffices to show that $L \subseteq \bar{V}^{Y}$. Indeed, let $x \in L$; since we have that $U$ is open in $\tau_{\alpha}$, we have that $x \in G$ such that $G$ is open in $\tau_{\alpha}, G \backslash\{x\}$ is Euclidean open and $G \subseteq U=V \cup L$. Arguing by contradiction, suppose that $x \in O, O$ is open in $Y$ and $O \cap V=\emptyset$ and observe that $G \cap O \backslash\{x\} \subseteq L$ and $G \cap O \backslash\{x\}$ is countable and non-empty (recall that $x$ is not isolated in $\left.\left(Y, \tau_{\alpha}\right)\right)$, contradicting lemma 3.5

We can now prove the following:

Claim 3: $W$ is dense in $[0,1]^{2}$ with the Euclidean topology iff $U$ is dense in $(X, \tau)$.

Proof of claim 3: Suppose that $U$ is dense in $(X, \tau)$; it is clear that $U$ is dense in $Y$, so $V$ is dense in $Y$. So $W$ is dense in $[0,1]^{2}$ with the Euclidean topology, since it contains $V$ and $Y$ is dense in $[0,1]^{2}$.

Conversely, suppose that $W$ is dense in $[0,1]^{2}$ with the Euclidean topology; we get that $V$ is dense in $Y$ (even in $[0,1]^{2}$ ) and so $U$ is dense in $Y$. An application of lemma 3.6 yields that $\bar{U}^{Y} \backslash \bar{U}^{\tau}$ is a countable set, so $X \backslash \bar{U}^{\tau}$ is a countable open set, so it is empty and we are done.

We now get that $B d_{Y}(U)=\bar{U}^{Y} \backslash \operatorname{Int}_{Y} U=\bar{V}^{Y} \backslash V=B d_{Y}(V)=B d(W) \cap Y$ and we conclude that $B d_{\tau}(U) \supseteq B d(W) \cap Y \backslash H$, where $H$ is a countable set, so to prove that $B d_{\tau}(U)$ is 1-dimensional, it suffices to show that $(B d(W) \cap Y, \tau\lceil B d(W) \cap Y)$ is 1-dimensional. This is so since we can apply the countable sum theorem in $\tau$ to the closed set $B d_{\tau}(U)$ and the countably many points of $H$. We have established that there exists $\alpha<\omega_{1}$ such that for all $\beta>\alpha, \operatorname{dim}\left(B d(W) \cap Y, \tau_{\beta}\lceil B d(W) \cap Y)=1\right.$. An application of lemma 3.6 finishes the proof.

\section{Related RESUlts AND QUestions}

In [10, Leibo showed that if $X$ is a closed image of a metric space, we have that $\operatorname{dim} X=\operatorname{Ind} X$. We have just seen (see [7]) that a continuous image of a separable metric space need not satisfy that $\operatorname{dim}=$ Ind, so the natural question (also due to Arhangel'skiı) is what happens when $X$ is a quotient of a separable metric space (For a theorem of Michael characterizing such spaces, see [7]). In particular, we do not know whether our space $X$ is a quotient of a separable metric space.

We also do not know how wide the possible gap between $\operatorname{dim}$ and ind $=$ Ind can be in a continuous image of a separable metric space. In particular, we do not know if $X$ is finite-dimensional or not.

A big question is whether $\mathrm{CH}$ is actually needed, i.e. whether such a space can be constructed in ZFC. In particular, what happens under $M A+\neg C H$ ?

We would like to thank the referee for many good and useful comments. In particular, it is the referee's observation that our space is the union of countably many separable metric spaces.

\section{REFERENCES}

1. A. V. Arhangel'skiǔ, A survey of some recent advances in general topology, old and new problems, Actes du congrès international des mathématiciens (Nice, 1970), Tome 2, GauthierVillars, 1971, pp. 19-26. MR 55:1269

2. B. Balcar and P. Simon, Disjoint refinement, Handbook of Boolean Algebras, Vol. 2 (J. D. Monk and R. Bonnet, eds.), North-Holland, 1989, pp. 333-386. CMP 21:00

3. R. Engelking, Dimension Theory, North Holland, 1978. MR 58:2753b

4. _ General Topology, Heldermann Verlag, 1989. MR 91c:54001 
5. V. V. Fedorčuk, Bicompacta without intermediate dimensions, Soviet Math. Dokl. 14 (1973), 1808-1811.

6. Compact spaces without canonically correct sets, Soviet Math. Dokl. 15 (No.5) (1974), 1272-1275.

7. G. Gruenhage, Generalized metric spaces, Handbook of Set-theoretic topology (K. Kunen and J. E. Vaughan, eds.), North-Holland, 1984, pp. 423-501. MR 86h:54038

8. _ Generalized metrics and metrization, Recent progress in general topology (M. Hušek and J. van Mill, eds.), North-Holland, 1992, pp. 237-274. CMP 93:15

9. K. Kuratowski, Une application des images de fonctions à la construction de certains ensembles singuliers, Mathematica 6 (1932), 120-123.

10. I. M. Leıbo, On the equality of dimensions for closed images of metric spaces, Soviet Math. Dokl. 15 (No. 3) (1974), 835-839.

11. K. Nagami, Dimension for $\sigma$-metric spaces, J. Math. Soc. Japan 23 (1971), 123-129. MR 44:4725

12. S. Oka, Dimension of finite unions of metric spaces, Math. Japon. 24 (1979), 351-362. MR 81d:54028

13. A. R. Pears, Dimension theory of general spaces, Cambridge University Press, 1975. MR 52:15405

14. S. Watson, The construction of topological spaces: Planks and resolutions, Recent progress in general topology (M. Hušek and J. van Mill, eds.), North-Holland, 1992, pp. 673-757. CMP $93: 15$

Department of Mathematics, York University, 4700 Keele St., North York, Ontario M3J 1P3 CANADA

Department of Mathematics, York University, 4700 Keele St., North York, Ontario M3J 1P3 CANADA

E-mail address: watson@mathstat.yorku.ca 